\title{
Symptomatisches Wirbelkörperhämangiom als Ursache eines Wurzelreizsyndroms
}

Symptomatische Hämangiome in Wirbelkörpern mit Ausbruch in den Spinalkanal sind ausgesprochene Raritäten, an allen Knochengeschwülsten haben sie höchstens einen Anteil von 1\%. Dagegen ist bei allen Röntgenuntersuchungen der Wirbelsäule mit asymptomatischen, zufällig röntgenologisch entdeckten Wirbelkörperhämangiomen mit einer Prävalenz von etwa $1 \% \mathrm{zu}$ rechnen (Freyschmidt J et al., Knochentumoren - Klinik, Radiologie, Pathologie 1988).

\section{Fallbeschreibung}

Die Vorstellung des 53-jährigen Patienten an der Neurologischen Ambulanz erfolgte wegen Verdachts auf Bandscheibenprotrusion bei Wurzelreizsyndrom L4/L5. Subjektiv bestanden seit etwa 6 Monaten lokalisierte Schmerzen und Faszikulationen.

Im Kernspintomogramm zeigte sich sowohl $\mathrm{T}_{1}$ - (Abb.1) als auch $\mathrm{T}_{2}$-gewichtet (Abb. 2) ein deutlicher Signalanstieg des 4. LWK, der zusätzlich inhomogen mit vereinzelt hypointensen Einlagerungen zur Darstellung gelangte.

Unmittelbar an der dorsalen Kante des Wirbelkörpers fand sich eine $\mathrm{T}_{1}$-gewichtet (Abb. 1) hypointense und $\mathrm{T}_{2}$-gewichtet (Abb. 2) hyperintense, glatt begrenzte, über eine zentrale Brücke mit dem Wirbelkörper in direkter Kontinuität stehende extradurale Raumforderung. $\mathrm{T}_{2}$-gewichtet bestand ein fast identischer Signalcharakter zum LWK 4.

Nach Kontrastmittelgabe (Abb. 3, 4) zeigte sich ein deutliches Enhancement der Läsion, die sich offensichtlich entlang des Kanals der Vena centralis in Richtung Spinalkanal ausbreitete und zu einer deutlichen Impression des Duralsacks führte. Axial (Abb.4) zeigte die Raumforderung zwei polsterförmige, paramedian gelegene Anteile mit rechtsseitiger Betonung. Die Neuroforamina wurden in diesem Segment von der Läsion tangiert, es bestand MR-tomographisch kein Hinweis auf eine Nervenwurzelirritation.

Eine angiographische oder histologische Abklärung wurde aufgrund des relativ

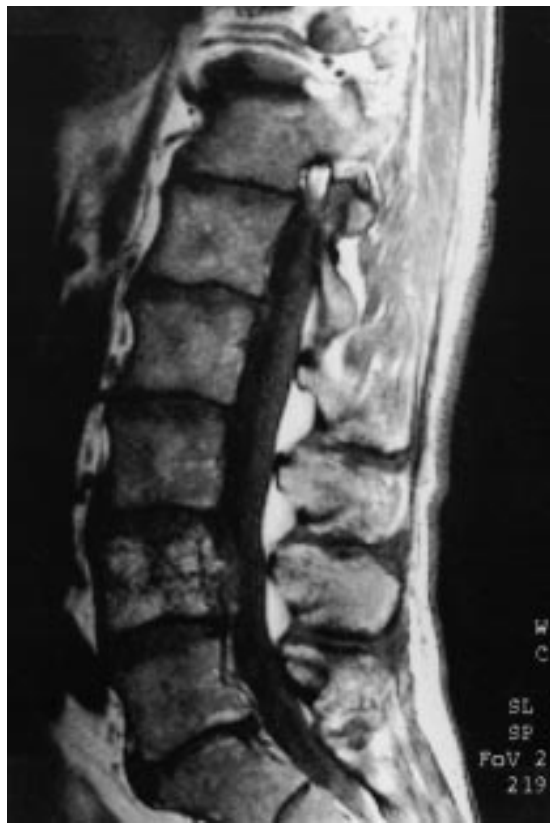

Abb. $1 \mathrm{~T}_{1}$-gewichtet, sagittal: Deutlicher Signalanstieg des LWK 4, der zusätzlich eine inhomogene Strukturalteration aufweist. An seiner dorsalen Kante findet sich eine hypointense extradurale, glatt begrenzte Raumforderung.

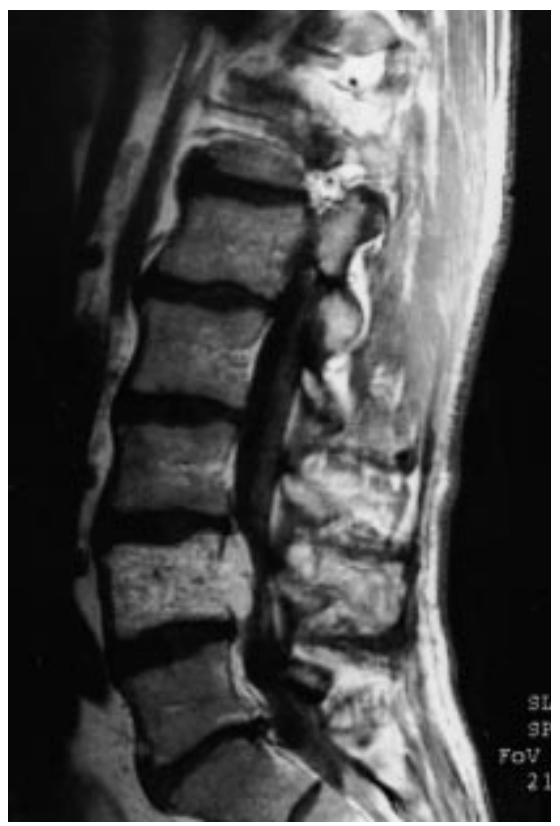

Abb. $3 \mathrm{~T}_{1}$-gewichtet, sagittal: Nach Kontrastmittelgabe findet sich ein deutliches Enhancement, wodurch sich die Läsion besser demarkiert.

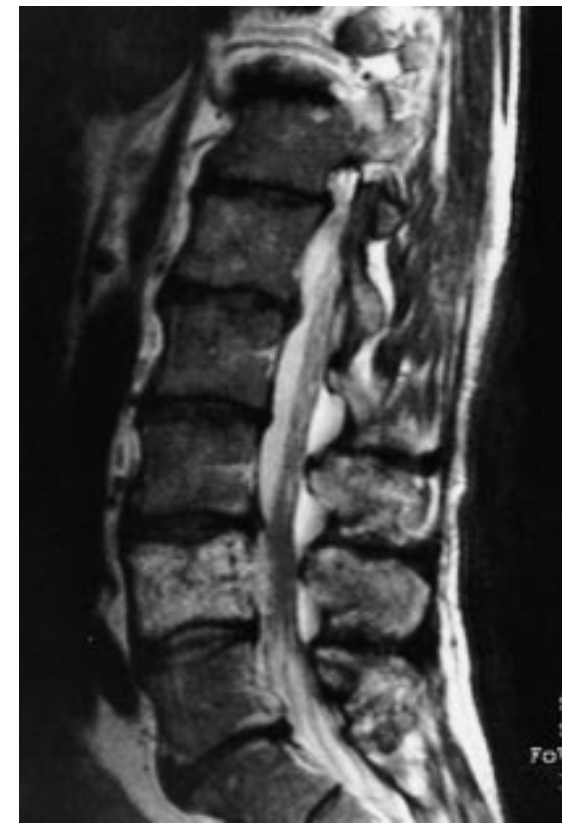

Abb. 2 Im $\mathrm{T}_{2}$-gewichteten Bild kommt die extradurale Raumforderung hyperintens zur Darstellung.

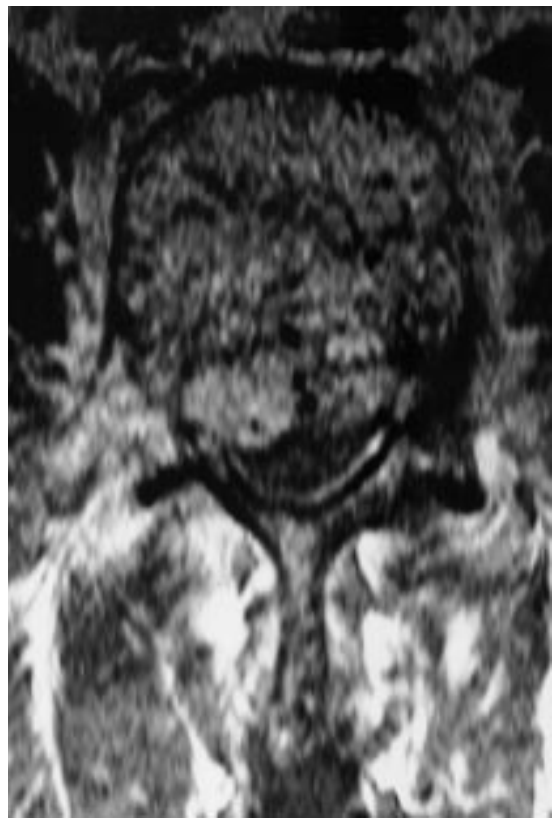

Abb. $4 \mathrm{~T}_{1}$-gewichtet, axial: Impression des Duralsacks durch die Raumforderung, die zwei polsterförmige, paramedian gelegene Anteile mit rechtsseitiger Betonung aufweist. 
typischen MR-tomographischen Erscheinungsbildes nicht durchgeführt.

Zumal über eine Verlaufsbeobachtung von nunmehr 8 Monaten keine progressive neurologische Symptomatik auftrat, wird der Patient weiterhin konservativ mit Schmerztherapie behandelt.

\section{Diskussion}

Bei klinisch symptomatischen und biopsierten Hämangiomen findet sich ein relativer Altersgipfel von ca. $32 \%$ in der 5. Lebensdekade (Dahlin DC, Bone tumors 1978). Die Prädilektionsstelle symptomatischer Wirbelkörperhämangiome ist die thorakale Wirbelsäule. Bis zum Auftreten von Symptomen besteht häufig bis zu einer mittleren Dauer von drei Jahren ein unauffälliger klinischer Verlauf. Die neurologische Symptomatik entwickelt sich meist progressiv nach vorausgehenden Schmerzen und Muskelspasmen.

Im Computertomogramm ist die Kombination von stärkeren fetthaltigen Arealen zwischen gröberen insgesamt rarefizierten vertikalen Spongiosatrabekeln mit einem starken Enhancement in den nichtfetthaltigen und -ossären Anteilen nach Kontrastmittelgabe spezifisch für das Vorliegen eines Wirbelkörperhämangioms.

Kernspintomographisch stellt sich das gewöhnliche Hämangiom im $\mathrm{T}_{1}$-gewichteten Bild signalintensiv dar, korrespondierend mit dem intertrabekulären Vakatfett.

Aggressivere und damit häufig symptomatische Hämangiome hingegen besitzen mehr Weichgewebsstroma und weniger Fett und verursachen somit eine Signalintensitätsminderung im $\mathrm{T}_{1}$-gewichteten Bild und Signalintensitätssteigerung bei der $\mathrm{T}_{2}$-Gewichtung. Nach Laredo und Braitinger (Laredo JD et al., Radiolgy 1990; 177: 467; Braitinger S et al., Fortschr Röntgenstr 1989; 151: 399) zeigen asymptomatische Wirbelhämangiome mit stark fetthaltigem Stroma(CT) und hoher Signalintensität im $\mathrm{T}_{1}$-gewichteten Bild keine oder nur diskrete Hypervaskularisation im Angiogramm, während agressive bzw. biologisch aktivere Hämangiome (wenig Fett im CT, Signalintensitätsminderung im MRT) eine stärkere Hypervaskularisation aufweisen. Letztere führen häufiger $\mathrm{zu}$ Kompressionsfrakturen und $\mathrm{zu}$ einem
Ausbruch aus dem Knochen mit Kompression des Spinalkanals.

Differentialdiagnostisch ist neben der Morphologie das MR-tomographische Verhalten von Hämangiomen relativ typisch und von sekundärblastomatösen Veränderungen $\left(\mathrm{T}_{1}\right.$ : hypointens; $\mathrm{T}_{2}$ : hyperintens) abzugrenzen. Wirklich intraossäre Lipome finden sich im Bereich der Wirbelsäule selten, fokale lipomatöse Ablagerungen sind der Morphologie von Hämangiomen ähnlich und ebenfalls hyperintens auf $\mathrm{T}_{1}$-gewichteten Sequenzen, zeigen in $\mathrm{T}_{2}$-Gewichtung allerdings eine dem Knochenmark ähnliche Signalintensität.

Die Empfehlung für das Management von Patienten mit ausschließlicher Schmerzsymptomatik besteht in jährlicher neurologischer und radiologischer Kontrolle sowie Schmerztherapie (Fox MW et al., J Neurosurg 1993; 78: 36-45).

Im Falle von therapierefraktären Schmerzen wird als Alternative eine Bestrahlung oder Embolisation vorge- schlagen. Bei Vorliegen eines progressiven neurologischen Defizits ist die präoperative Angiographie mit Embolisation bzw. eine chirurgische Dekompression und zusätzliche postoperative Radiatio bei subtotaler Tumorentfernung angezeigt. Die Wahl des Verfahrens und der Grad der chirurgischen Invasivität richten sich nach Lokalisation und Ausmaß des Wirbelkörperbefalls und/ oder Weichteilanteils und dem neurologischen Status. Vielfach wird, je nach Ausmaß und Lokalisation der Läsion und Durchblutungsgrad auch die präoperative Embolisation zur Reduktion des intraoperativen Blutverlustes empfohlen, vor allem bei geplanter subtotaler oder totaler Wirbelkörperexzision.

Die Prognose des Hämangioms ist insgesamt gut, auch im Falle eines Ausbruchs in den Spinalkanal, vorausgesetzt, solche Hämangiome sind einer Operation ausreichend zugänglich.

S. Huber, A. Kladosek, H. Czembirek, Wien, Austria 